\title{
氮肥和种植密度对达乌里胡枝子的生长与生物固氮 的影响
}

\author{
王银柳 1,2 耿倩倩 ${ }^{1,2}$ 黄建辉 $1,2^{*}$ 王常慧 ${ }^{1}$ 李 䂞 1,2 哈斯木其尔 ${ }^{1,2}$ 牛国祥 1,2 \\ ${ }^{1}$ 中国科学院植物研究所植被与环境变化国家重点实验室, 北京 $100093 ;{ }^{2}$ 中国科学院大学资源与环境学院, 北京 100049
}

\begin{abstract}
摘 要 氮供给和种植密度是影响植物生长的两个重要因素。豆科植物因其生物固氮能力而在受到氮限制的生态系统中具有 重要作用, 氮含量增加促进植物生长的同时也会抑制豆科植物的生物固氮能力, 种植密度会通过种内竞争影响豆科植物的生 长和生物固氮能力, 然而少有研究关注氮肥添加和种植密度对豆科植物生长和生物固氮能力的影响。该研究以达乌里胡枝子 (Lespedeza davurica) 为研究对象, 通过温室盆栽实验, 探究氮肥和种植密度对其生长和生物固氮的影响。实验设置 4 个氮添加 水平 $\left(0 、 5 、 10 、 20 \mathrm{~g} \cdot \mathrm{m}^{-2} \cdot \mathrm{a}^{-1}\right)$ 和 3 种种植密度(1、3、6 Ind. $\operatorname{pot}^{-1}$, 约32、96、192 Ind. $\left.\cdot \mathrm{m}^{-2}\right)$ 。结果发现: 1)施肥和密度增加均影 响了达乌里胡枝子的生长。叶片碳 $(\mathrm{C})$ 、氮 $(\mathrm{N})$ 含量、净光合速率随施氮量增加而增加, 氮添加也促进了植物的生长, 当施氮 量为 $10 \mathrm{~g} \cdot \mathrm{m}^{-2} \cdot \mathrm{a}^{-1}$ 时植物产量达到最大。叶片 $\mathrm{C} 、 \mathrm{~N}$ 含量、净光合速率随种植密度增加而下降, 密度增加可以促进每盆的总生 物量, 但对单个植株的生长有负效应。2)氮肥对根瘤形成有抑制作用, 但种植密度增加会缓解氮肥对生物固氮能力带来的“氮 阻遏”。该实验条件下, 当施氮量为 $10 \mathrm{~g} \cdot \mathrm{m}^{-2} \cdot \mathrm{a}^{-1}$, 种植密度为 3 Ind. $\operatorname{pot}^{-1}$, 或施氮量为 $5 \mathrm{~g} \cdot \mathrm{m}^{-2} \cdot \mathrm{a}^{-1}$, 种植密度为 6 Ind. pot $^{-1}$ 时, 能 最大程度发挥“施氮增产”和种植密度缓解“氮阻遏”的作用。氮添加降低了达乌里胡枝子的根瘤生物量和对根瘤形成的投资 (根瘤生物量占总生物量的比例), 从而抑制达乌里胡枝子的生物固氮。种植密度增加导致达乌里胡枝子因种内竞争增加而使 资源获取受限, 从而增加对根瘤的投资和根瘤生物量来获得更多来自大气中的氮。3)结构方程结果显示, 氮肥和种植密度通 过直接或间接作用, 解释了 $64 \%$ 的达乌里胡枝子生物量变化和 $42 \%$ 的根瘤生物量变化。上述结果表明合理优化豆科植物的施 肥量和种植密度可能对人工草地种植以及退化草地恢复管理具有重要意义。
\end{abstract}

关键词 氮肥添加；种植密度；生物量；根瘤生物量；根瘤投资；生物固氮

王银柳, 耿倩倩, 黄建辉, 王常慧, 李否, 哈斯木其尔, 牛国祥 (2021). 氮肥和种植密度对达乌里胡枝子的生长与生物固氮的影响. 植物生态学报, 45, 13-22. DOI: $10.17521 /$ cjpe.2020.0185

\section{Effects of nitrogen addition and planting density on the growth and biological nitrogen fixa- tion of Lespedeza davurica}

WANG Yin-Liu ${ }^{1,2}$, GENG Qian-Qian ${ }^{1,2}$, HUANG Jian-Hui ${ }^{1,2^{*}}$, WANG Chang-Hui ${ }^{1}$, LI Lei ${ }^{1,2}$, HASI Muqier ${ }^{1,2}$, and NIU Guo-Xiang ${ }^{1,2}$

${ }^{1}$ State Key Laboratory of Vegetation and Environmental Change, Institute of Botany, Chinese Academy of Sciences, Beijing 100093, China; and ${ }^{2}$ College of Resources and Environment, University of Chinese Academy of Sciences, Beijing 100049, China

\begin{abstract}
Aims Nitrogen (N) supply and planting density are two important factors influencing plant growth. Legumes are important to ecosystem $\mathrm{N}$ input because of their capacity of biological $\mathrm{N}_{2}$ fixation (BNF). Increasing atmospheric $\mathrm{N}$ deposition may promote the growth of leguminous plants, but it may also inhibit strongly their BNF capacity. Planting density can also influence the growth and BNF capacity of legumes due to intraspecific competition. However, few studies up to date have focused on the effects of $\mathrm{N}$ fertilization and planting density on the growth and BNF capacity of legumes. In this study, we aimed to explore the potentially interactive effects of $\mathrm{N}$ fertilization and planting density on the growth and BNF capacity of Lespedeza davurica, a leguminous plant species which is widely distributed throughout the northern China grasslands.
\end{abstract}

Methods A pot experiment was conducted in a greenhouse. The experiment contained four levels of $\mathrm{N}$ addition $\left(0,5,10,20 \mathrm{~g} \cdot \mathrm{m}^{-2} \cdot \mathrm{a}^{-1}\right)$ by $\mathrm{NH}_{4} \mathrm{NO}_{3}$ fertilizer and three levels of planting densities $\left(1,3,6 \mathrm{Ind} \cdot\right.$ pot $^{-1}$, i.e. 32,96 , 192 Ind. $\left.\cdot \mathrm{m}^{-2}\right)$. 
Important findings 1) Our results showed that both $\mathrm{N}$ addition and planting density could impact the biomass production of $L$. davurica. $\mathrm{N}$ addition increased plant leaf carbon $(\mathrm{C})$ and $\mathrm{N}$ contents and leaf-level net photosynthetic rate. Besides, $\mathrm{N}$ addition also stimulated the plant growth at both pot and individual levels, and the yield reached maximum at $\mathrm{N}$ addition of $10 \mathrm{~g} \cdot \mathrm{m}^{-2} \cdot \mathrm{a}^{-1}$. Increasing planting density decreased leaf $\mathrm{C}$ and $\mathrm{N}$ contents, leaf-level net photosynthetic rate, and individual growth, but increased total biomass in each pot. 2) Nitrogen addition reduced the capacity of BNF of L. davurica, while increasing planting density could weaken this suppression effect to some extent. The combination of $\mathrm{N}$ addition of $10 \mathrm{~g} \cdot \mathrm{m}^{-2} \cdot \mathrm{a}^{-1}$ and planting density of $3 \mathrm{Ind} \cdot \mathrm{pot}^{-1}$ or $\mathrm{N}$ addition of $10 \mathrm{~g} \cdot \mathrm{m}^{-2} \cdot \mathrm{a}^{-1}$ and planting density of $6 \mathrm{Ind} \cdot \operatorname{pot}^{-1}$ could maximize the effects of $\mathrm{N}$ application on individual yield and the effects of increasing planting density on the alleviation of BNF suppression. Nitrogen addition suppressed the BNF of $L$. davurica through reducing plant investment to nodulation and nodule biomass production. The intraspecific competition and resource limitation caused by increasing planting density led to improvement in the investment to nodulation and nodule growth. 3) Structural equation model analyses showed that $\mathrm{N}$ addition and planting density combined explained variations in the plant biomass and nodule production either directly or indirectly by $64 \%$ and $42 \%$, respectively. The results indicate that it is important to optimize the amount of fertilizer application and appropriate planting density when considering plantation and management of artificial and degraded grasslands.

Key words $\mathrm{N}$ addition; planting density; biomass; nodule biomass; investment to nodulation; biological nitrogen fixation

Wang YL, Geng QQ, Huang JH, Wang CH, Li L, Hasi M, Niu GX (2021). Effects of nitrogen addition and planting density on the growth and biological nitrogen fixation of Lespedeza davurica. Chinese Journal of Plant Ecology, 45, 13-22. DOI: $10.17521 /$ cjpe. 2020.0185

氮 $(\mathrm{N})$ 是植物生长和发育所必需的重要养分元 素(LeBauer \& Treseder, 2008), 同时也是陆地生态 系统生产力的限制性元素(Vitousek \& Howarth, 1991; Elser et al., 2007)。自工业革命以来, 人类活动 导致的大气氮沉降日益增加(Galloway et al., 2004, 2008), 这在缓解生态系统的氮限制的同时也改变 了生态系统的结构和功能(Pardo et al., 2011; Midolo et al., 2019)。对豆科植物而言, 由于其生物固氮功 能在受到氮限制的生态系统中占有重要地位 (Vitousek et al., 2013), 但通过共生固氮获取大气中 的氮是一个高耗能的过程(Gutschick, 1981), 大气氮 沉降的增加以及草原管理中氮肥的施用提高了土壤 中可利用氮的含量(潘庆民等, 2005; Liu et al., 2013), 这为豆科植物提供了一个相对“便宜”的氮源, 豆科 植物可能会调整其对氮的利用方式以及对生物固氮 的投资来保持其在群落中的竞争能力(Menge et al., 2009)。目前关于豆科植物对氮响应的研究已经有很 多, 但不同物种的生长和生物固氮对氮水平的响应 并不一致(Guinet et al., 2018), 例如Regus等(2017) 发现氮沉降显著降低了豆科植物Acmispon strigosus 的根瘤数量和生物量, 而Drake (2011)的研究表明 施氮会显著促进豆科植物生长, 同时对生物固氮没 有抑制作用。因此, 结合具体豆科植物生物固氮和 生长情况对土壤氮供给水平的响应, 可以评价豆科
植物在氮沉降或草原施氮管理背景下群落中重要性 的变化。

密度依赖性引起的植株正或负相互作用普遍存 在于植物种群中, 选择合适的种植密度是建植和管 理人工草地的关键(Japhet et al., 2009; 张炜平和王 根轩, 2010)。低密度下单个植株的生长空间较大, 资源充足, 但群体产量受到个体数量限制。相反, 密 度增加提高群体产量的同时也会导致群落内资源竞 争增加(Weiner et al., 2001), 影响单个植株的生长 发育和干物质积累(Chu et al., 2008; 列志昒等, 2016), 并改变生物量分配(赵宏魁等, 2016)。

人工草地以及农业管理中往往将施肥和种植密 度结合起来以实现增产和效益最大化。对大豆 (Glycine max)的研究表明, 大豆的产量随密度和肥 力的增加而增加, 高产是由中密度、高密度和中肥 量、高肥量相结合实现的(杨继学等, 2012)。张明聪 等(2018)发现相同密度下施加少量基肥可以促进红 小豆(Vigna umbellata)根瘤的形成, 后期追肥可以延 长叶片的功能期, 而前期一次性施加基肥则导致营 养体过大，同时会抑制根瘤的形成，导致早衰和减 产; 相同氮添加水平下单株植物的干物质积累随密 度增加而下降。虽然关于种植密度和施肥处理对豆 科植物影响的研究已有不少, 但主要集中在大豆等 豆科作物中, 而且并未充分考虑生物固氮的效果,

www.plant-ecology.com 
对于草原豆科物种的相关研究仍然存在不足。

达乌里胡枝子 (Lespedeza davurica) 是豆科蝶形 花亚科胡枝子属(Lespedeza)的一种多年生半灌木状 草本植物, 是改良干旱、半干旱区退化草地和人工 放牧地的优良品种(赵祥等, 2009; 段东平等, 2012)。 研究表明, 达乌里胡枝子的种子发芽率较高, 具有 抗氧化防御系统来应对干旱以及UV-B辐射等逆境 胁迫(马彦军等, 2010; 杜润峰, 2012), 常为草原群 落的次优势种和伴生种, 在受火烧以及森林砍伐严 重的地区，常常形成优势种(陈默君等，1997), 并且 在全球气候变暖的背景下, 达乌里胡枝子的种群生 态幅在扩大(程杰等, 2011)。虽然关于达乌里胡枝子 生长特性以及应对环境变化的研究已有不少, 但鲜 有研究关注达乌里胡枝子的生物固氮功能以及多种 管理措施下达乌里胡枝子的生长情况。因此, 本研 究通过盆栽实验探究不同水平的氮肥添加量和种植 密度对达乌里胡枝子的生长和生物固氮的影响, 旨 在寻找科学的氮肥添加量和种植密度, 为人工草地 建设中达乌里胡枝子的合理利用提供科学依据。

\section{1 材料和方法}

\section{1 实验设计}

实验于2019年5-8月在中国科学院植物研究所 的实验温室内进行。温室温度维持在 $(25 \pm 5){ }^{\circ} \mathrm{C}$, 相 对湿度为 $20 \%$ 。达乌里胡枝子的种子为山西农业大 学牧站草学实验室种子资源库提供的“晋农 1 号” (千 粒质量 $1.90 \mathrm{~g}$, 发芽率为 $50.80 \%$ )。挑选籽粒饱满的 达乌里胡枝子种子在培养血中浸泡催芽(马彦军等, 2010) 后, 移栽到塑料花盆中 (直径 $20 \mathrm{~cm}$, 高度 $25 \mathrm{~cm}$ )。培养基质为取自内蒙古多伦县典型草原的 表层土壤 $(0-20 \mathrm{~cm})$, 每盆装土约 $5.0 \mathrm{~kg}$ 。为保证实验 期间土壤其他养分充足, 实验之前每盆土壤中加入 $\mathrm{Ca}\left(\mathrm{H}_{2} \mathrm{PO}_{4}\right)_{2} 0.41 \mathrm{~g} 、 \mathrm{~K}_{2} \mathrm{SO}_{4} 0.56 \mathrm{~g}$ 和 $\mathrm{MgSO}_{4} 2.05 \mathrm{~g}$, 并 充分混匀。

本实验参考我国大气氮沉降量 $\left(2.04 \mathrm{~g} \cdot \mathrm{m}^{-2} \cdot \mathrm{a}^{-1}\right.$, Yu et al., 2019) 以及其他学者类似研究的设计(张晓 红等, 2007; 关少波, 2017), 采用随机区组设计, 设 置氮肥水平和种植密度 2 个因素。其中, 氮肥施氮水 平为 $0 、 5 、 10$ 和 $20 \mathrm{~g} \cdot \mathrm{m}^{-2} \cdot \mathrm{a}^{-1}$ 共 4 个水平, 所施氮肥为 $\mathrm{NH}_{4} \mathrm{NO}_{3}$ 。种植密度处理为 $1 、 3 、 6$ Ind. pot $^{-1}$, 约 32 、 96、 192 Ind. $\cdot \mathrm{m}^{-2}$ 共 3 个水平。两个因素共有 12 种处 理, 每种处理 5 次重复, 共计 60 盆。移栽 2 周后按设定
密度对移栽的达乌里胡枝子进行间苗，并在实验的 第4周进行氮肥添加处理。

\section{2 测定方法}

实验的第11周于每天9:00-11:30采用LI-6800便 携式光合仪(LI-COR, Lincoln, USA) 人工补光到 $1200 \mu \mathrm{mol} \cdot \mathrm{m}^{-2} \cdot \mathrm{s}^{-1}$, 每株植物选择 3 片成熟的叶片测 定净光合速率 $\left(P_{\mathrm{n}}\right)$, 之后将叶片取下扫描测定叶面 积。生物量测定于实验的第12周结束后进行, 收割 前测定每盆里单个植株的株高, 从地上部分采集植 物10-20片新鲜成熟叶片单独装入信封。剩余地上部 分装入另一信封, $105{ }^{\circ} \mathrm{C}$ 杀青 $30 \mathrm{~min}$ 后, 在 $65{ }^{\circ} \mathrm{C}$ 条 件下烘干至恒质量。分别测定两部分的生物量, 其 和即为地上生物量。单独采集的叶片粉碎后用元素 分析仪 (vario EL III, Elementar Analysensysteme $\mathrm{GmbH}$, Hesse, Germany)测定叶片C、N含量。地下 部分清洗干净后摘取根瘤并计数, 根系与根瘤烘干 后分别称质量, 根系与根瘤的生物量之和为地下生 物量。

\section{3 数据处理与计算}

总生物量为地上生物量与地下生物量之和, 平 均生物量为总生物量除以对应的植株数目。根冠比 为地下生物量与地上生物量的比值。

采用相对邻株效应指数 (RNE) 确定不同密度处 理下达乌里胡枝子的种内竞争强度(Chu et al., 2008)。该指数计算公式为:

$$
R N E=\frac{\bar{x}_{1}-\bar{x}_{0}}{\bar{x}_{\max }}
$$

式中, $\bar{x}_{1}$ 和 $\bar{x}_{0}$ 分别为有和无相邻个体时种群内个体 的平均生物量, $\bar{x}_{\text {max }}$ 是两者之间的最大值。RNE值的 范围为 $-1-1$, 为负值时表示植株间为负相互作用关 系, 即竞争关系, 且绝对值越大表示竞争越强; RNE 为正值时表示为正相互作用关系, 即促进关系, 且 绝对值越大表示植株间促进作用越强。

根瘤生物量的响应比 $(R R)$ 表示不同处理下达乌 里胡枝子根瘤生物量相对于对照(1 Ind. pot $^{-1}, 0 \mathrm{~g}$ $\mathrm{N} \cdot \mathrm{m}^{-2} \cdot \mathrm{a}^{-1}$ ) 的变化(励玮等, 2018), 计算公式为:

$$
R R=\frac{\bar{x}_{\mathrm{T}}}{\bar{x}_{\mathrm{C}}}
$$

式中, $\bar{x}_{\mathrm{T}}$ 为处理条件下每盆达乌里胡枝子的根瘤生 物量, $\bar{x}_{\mathrm{C}}$ 为对照条件下的根瘤生物量。 $R R>1$ 为正 响应, 即根瘤的生物量增加; $R R<1$ 为负相应, 根瘤 的生物量与对照相比减少。 
另外用根瘤投资(investment to nodulation)表示 根瘤生物量相对于总生物量所占的比例(Batterman et al., 2013)。

分析前对数据进行正态性和方差同质性的检验, 对不满足条件的数据进行了对数转换。采用双因素 方差分析来分析氮肥和种植密度对达乌里胡枝子的 总生物量, 叶片 $\mathrm{C} 、 \mathrm{~N}$ 含量, 净光合速率, 根瘤投资 等指标的影响。采用Duncan多重比较方法进行显著 性检验 $(p<0.05)$ 。以上数据分析均采用 $\mathrm{R}$ 软件 (Version 3.6.2)进行。采用AMOS 21.0软件构建结构 方程模型(SEM), 分析氮添加和种植密度如何通过 根瘤生物量、净光合速率和叶片特征影响达乌里胡 枝子的生长和生物固氮。在构建 SEM之前对数据进 行标准化处理, 并且对 3 个叶片特征指标(叶片 C、N 含量、叶面积)进行主成分分析提取 PC1, PC1的解释 量为 $52.28 \%$ 。卡方检验显著性 $p>0.05$ 的模型可被 接受。

\section{2 结果}

\section{1 生长量对氮肥和种植密度的响应}

氮肥添加对植物个体株高无显著影响 $\left(F_{\mathrm{N}(3,48)}=\right.$ $1.47, p>0.05)$, 但对总生物量 $\left(F_{\mathrm{N}(3,48)}=11.56, p<\right.$ $0.001)$ 、总地上生物量 $\left(F_{\mathrm{N}(3,48)}=11.60, p<0.001\right)$ 、 总地下生物量 $\left(F_{\mathrm{N}(3,48)}=8.70, p<0.001\right)$ 、平均地上 生物量 $\left(F_{\mathrm{N}(3,48)}=7.73, p<0.001\right)$ 、平均地下生物量 $\left(F_{\mathrm{N}(3,48)}=5.43, p<0.01\right)$ 以及根冠比 $\left(F_{\mathrm{N}(3,48)}=3.32, p\right.$ $<0.05)$ 均存在显著影响 $(p<0.05)$, 除根冠比外其他 指标均随施氮量的增加呈现上升的趋势。种植密度
处理则对所有的生长指标均有显著作用，其中总生 物量 $\left(F_{\mathrm{d}(2,48)}=69.67, p<0.001\right)$ 、总地上生物量 $\left(F_{\mathrm{d}(2,48)}=61.95, p<0.001\right)$ 、总地下生物量 $\left(F_{\mathrm{d}(2,48)}=\right.$ $32.10, p<0.001)$ 以及根冠比 $\left(F_{\mathrm{d}(2,48)}=13.95, p<\right.$ $0.001)$ 均随种植密度增加呈增加趋势, 而株高 $\left(F_{\mathrm{d}(2,48)}\right.$ $=15.90, p<0.001)$ 、平均地上生物量 $\left(F_{\mathrm{d}(2,48)}=\right.$ $168.03, p<0.001)$ 和平均地下生物量 $\left(F_{\mathrm{d}(2,48)}=10.69\right.$, $p<0.001$ )则随密度增加呈现降低趋势(表1)。

\section{2 叶片 $\mathrm{C} 、 \mathrm{~N}$ 含量与净光合速率对氮肥和种植密 度的响应}

氮肥添加和种植密度处理均显著影响达乌里胡 枝子叶片的 $\mathrm{C}\left(F_{\mathrm{N}(3,48)}=3.77, F_{\mathrm{d}(2,48)}=59.55, p<\right.$ $0.01) 、 \mathrm{~N}$ 含量 $\left(F_{\mathrm{N}(3,48)}=11.74, F_{\mathrm{d}(2,48)}=39.94, p<0.05\right)$ 和叶片净光合速率 $\left(F_{\mathrm{N}(3,941)}=5.38, F_{\mathrm{d}(2,941)}=26.52\right.$, $p<0.001)$, 二者交互作用对叶片 $\mathrm{C}\left(F_{\mathrm{N} \times \mathrm{d}(6,48)}=0.98\right.$, $p>0.05) 、 \mathrm{~N}$ 含量 $\left(F_{\mathrm{N} \times \mathrm{d}(6,48)}=0.61, p>0.05\right)$ 无显著影 响(表1; 图1A), 但对叶片净光合速率存在极显著影 响 $\left(F_{\mathrm{N} \times \mathrm{d}(6,941)}=44.64, p<0.001\right)$ 。氮添加显著提高了 叶片 $\mathrm{N}$ 含量, 但随着种植密度的增加叶片 $\mathrm{N}$ 含量显 著下降。氮添加提高了各密度水平下的叶片净光合 速率和叶片 C含量(表1; 图1B), 但叶片的 C含量随 种植密度增加显著降低(表 1$)$ 。在种植密度为 1 和 6 Ind. pot $^{-1}$ 的处理下, 净光合速率在施氮量为 10 $\mathrm{g} \cdot \mathrm{m}^{-2} \cdot \mathrm{a}^{-1}$ 时达到最高, 之后随施氮量增加而呈下降 趋势, 而 3 Ind. pot $^{-1}$ 处理下叶片净光合速率随施氮 量的增加而增加。在 $0-10 \mathrm{~g} \cdot \mathrm{m}^{-2} \cdot \mathrm{a}^{-1}$ 施氮水平下密度 为 1 Ind. $\operatorname{pot}^{-1}$ 的处理叶片净光合速率最高, 在施氮 量达到 $20 \mathrm{~g} \cdot \mathrm{m}^{-2} \cdot \mathrm{a}^{-1}$ 时净光合速率最低。

表1 不同氮肥和种植密度处理下达乌里胡枝子的生长指标(平均值土标准误)

Table 1 Growth indexes of Lespedeza davurica under different levels of nitrogen addition and planting density treatments (mean $\pm S E$ )

\begin{tabular}{|c|c|c|c|c|c|c|c|c|c|}
\hline $\begin{array}{l}\text { 处理 } \\
\text { Treatment }\end{array}$ & $\begin{array}{l}\text { 水平 } \\
\text { Level }\end{array}$ & $\begin{array}{c}\text { 株高 } \\
\text { Height (cm) }\end{array}$ & $\begin{array}{c}\text { 总生物量 } \\
\text { Total } \\
\text { biomass (g) }\end{array}$ & $\begin{array}{c}\text { 总地上生物量 } \\
\text { Total } \\
\text { aboveground } \\
\text { biomass (g) }\end{array}$ & $\begin{array}{c}\text { 总地下生物量 } \\
\text { Total } \\
\text { belowground } \\
\text { biomass (g) }\end{array}$ & $\begin{array}{c}\text { 平均地上生物量 } \\
\text { Average } \\
\text { aboveground } \\
\text { biomass }\left(\mathrm{g} \cdot \mathrm{Ind} .^{-1} \text { ) }\right.\end{array}$ & $\begin{array}{c}\text { 平均地下生物量 } \\
\text { Average } \\
\text { belowground } \\
\text { biomass }\left(\mathrm{g} \cdot \text { Ind. }^{-1} \text { ) }\right.\end{array}$ & $\begin{array}{l}\text { 根冠比 } \\
\text { Root:shoot } \\
\text { ratio }\end{array}$ & $\begin{array}{c}\text { 叶片C含量 } \\
\text { Leaf C } \\
\text { concentration } \\
(\%)\end{array}$ \\
\hline \multirow{4}{*}{$\begin{array}{l}\text { 施氮 } \\
\mathrm{N} \text { addition } \\
\left(\mathrm{g} \cdot \mathrm{m}^{-2} \cdot \mathrm{a}^{-1}\right)\end{array}$} & 0 & & $11.79 \pm 1.02^{\mathrm{b}}$ & $4 \pm 0.72^{\mathrm{c}}$ & $2.84 \pm 0.32^{b}$ & $3.61 \pm 0.47^{\mathrm{b}}$ & $1.06 \pm 0.13^{b}$ & & $45.26 \pm 0.34^{b}$ \\
\hline & 5 & 49 & $.43 \pm 1.02^{\mathrm{a}}$ & $140 \mathrm{~s}$ & a & $3+0$ & $2^{\mathrm{a}}$ & $2^{\mathrm{a}}$ & $.35^{\mathrm{a}}$ \\
\hline & 10 & $62.56 \pm 2.43^{\mathrm{ns}}$ & $15.76 \pm 1.04^{\mathrm{a}}$ & $11.99 \pm 0.77^{\mathrm{a}}$ & $3.76 \pm 0.32^{\mathrm{a}}$ & $5.20 \pm 0.80^{\mathrm{a}}$ & $1.60 \pm 0.25^{\mathrm{a}}$ & $0.31 \pm 0.02^{\mathrm{b}}$ & $45.98 \pm 0.32^{\mathrm{a}}$ \\
\hline & 20 & $63.32 \pm 2.61^{\mathrm{ns}}$ & $15.37 \pm 1.02^{\mathrm{a}}$ & $11.52 \pm 0.7^{\mathrm{ab}}$ & $3.86 \pm 0.33^{\mathrm{a}}$ & $4.91 \pm 0.67^{\mathrm{a}}$ & $1.51 \pm 0.17^{\mathrm{a}}$ & $0.33 \pm 0.01^{\mathrm{ab}}$ & $45.95 \pm 0.26^{\mathrm{a}}$ \\
\hline \multirow{3}{*}{$\begin{array}{l}\text { 密度 } \\
\text { Planting } \\
\text { density } \\
\text { (Ind.·pot }{ }^{-1} \text { ) }\end{array}$} & 1 & $11.53 \pm 4.43$ & $10.01 \pm 0.62^{\mathrm{b}}$ & $7.80 \pm 0.45^{\mathrm{b}}$ & $2.20+5,8$ & $1.01 \pm 0.43$ & $2.20 \pm 0.19^{\mathrm{a}}$ & $0.28 \pm 0.01^{\mathrm{b}}$ & $46.29 \pm 0.09^{\mathrm{a}}$ \\
\hline & 3 & $61.56 \pm 1.60^{\mathrm{b}}$ & $16.54 \pm 0.62^{\mathrm{a}}$ & $12.20 \pm 0.46^{\mathrm{a}}$ & $4.34 \pm 0.19^{\mathrm{a}}$ & $4.07 \pm 0.15^{\mathrm{b}}$ & $1.45 \pm 0.06^{\mathrm{b}}$ & $0.36 \pm 0.01^{\mathrm{a}}$ & $46.69 \pm 0.24^{\mathrm{a}}$ \\
\hline & 6 & $57.53 \pm 1.42^{\mathrm{b}}$ & $17.50 \pm 0.55^{\mathrm{a}}$ & $12.94 \pm 0.38^{\mathrm{a}}$ & $4.56 \pm 0.25^{\mathrm{a}}$ & $2.16 \pm 0.06^{\mathrm{c}}$ & $0.76 \pm 0.04^{\mathrm{c}}$ & $0.35 \pm 0.02^{\mathrm{a}}$ & $44.41 \pm 0.13^{\mathrm{b}}$ \\
\hline & & & ** & $* * *$ & $* * *$ & $* * *$ & $* * *$ & $* * *$ & ns \\
\hline
\end{tabular}

同列不同小写字母表示氮或密度处理下差异显著 $(p<0.05), \mathrm{ns}$ 表示差异不显著 $(p>0.05)$ 。,$p<0.05 ; * *, p<0.01 ; * * *, p<0.001$ 。

Different lowercase letters in the same column indicate significant difference among $\mathrm{N}$ addition levels or density treatments at $p<0.05$ level, while ns indicates no significant difference. *, $p<0.05 ; * *, p<0.01 ; * * *, p<0.001$.

www.plant-ecology.com 


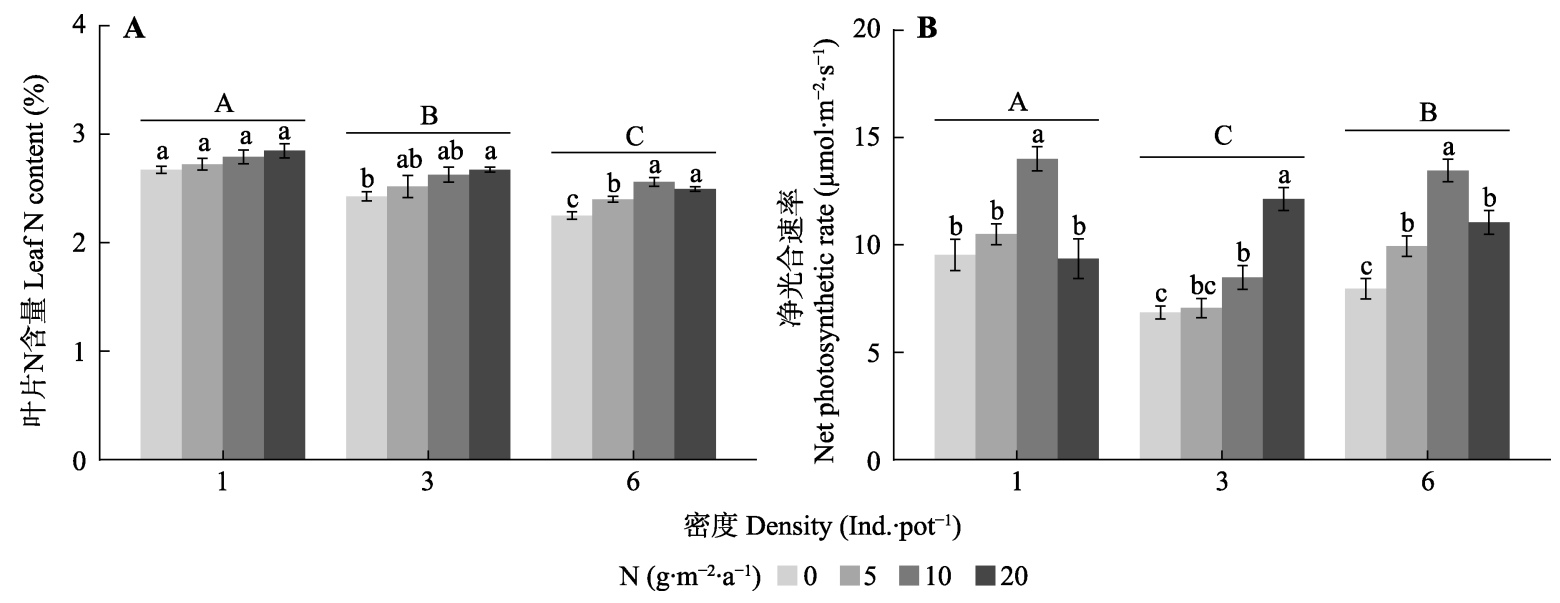

图1 不同处理下达乌里胡枝子的叶片 $\mathrm{N}$ 含量(A)和净光合速率(B)的变化(平均值土标准误)。不同小写字母表示同一密度下不同 施氮量间差异显著 $(p<0.05)$, 不同大写字母表示不同密度下差异显著 $(p<0.05)$ 。ns, $p>0.05 ; *, p<0.05 ; * *, p<0.01 ; * * *, p<$ 0.001 。

Fig. 1 Changes in leaf nitrogen $(\mathrm{N})$ concentration $(\mathbf{A})$ and net photosynthetic rate $(\mathbf{B})$ of Lespedeza davurica under different treatments (mean $\pm S E$ ). Different lowercase letters indicate significant difference among $\mathrm{N}$ addition treatments under the same density at $p<0.05$ level, while different uppercase letters indicate significant difference among density treatments at $p<0.05$ level. ns, $p>0.05$; $*, p<0.05 ; * *, p<0.01 ; * * *, p<0.001$.

\section{3 相对邻株效应指数对氮肥和种植密度的响应}

相对邻株效应指数反映了植株间的竞争强度, 结果表明种植密度均极显著影响达乌里胡枝子的种 内竞争强度 $\left(F_{\mathrm{d}(1,32)}=57.73, p<0.001\right)$, 氮肥添加对 种内竞争强度无显著影响 $\left(F_{\mathrm{N}(3,32)}=1.51, p>0.05\right)$ 且 交互作用不显著 $\left(F_{\mathrm{N}(3,32)}=1.51, p>0.05\right)$ (图2)。在无 氮肥添加情况下, 增加种植密度对种内竞争强度无 显著影响; 氮肥添加后种植密度的增加会导致达乌 里胡枝子的种内竞争的增加。

\section{4 达乌里胡枝子生物固氮对氮肥和种植密度的} 响应

研究结果表明, 氮肥添加 $\left(F_{\mathrm{N}(3,48)}=53.25, p<\right.$ $0.05)$ 与种植密度 $\left(F_{\mathrm{d}(2,48)}=26.02, p<0.001\right)$ 均对根瘤 的形成有显著影响, 且两者间存在极显著 $\left(F_{\mathrm{N} \times \mathrm{d}(6,48)}\right.$ $=15.01, p<0.001)$ 的交互作用(图3A)。氮添加显著 降低了达乌里胡枝子根瘤生物量, 增加种植密度则 可以提高根瘤的生物量。两者的交互作用体现为密 度增加降低了氮肥的效应, 具体表现为单株种植情 况下, 当氮添加量达到 $5 \mathrm{~g} \cdot \mathrm{m}^{-2} \cdot \mathrm{a}^{-1}$ 时几乎无根瘤形 成(根瘤数量为 $0.57 \pm 0.09$ ), 而在增加种植密度的情 况下各施氮水平均有根瘤生成。3 和 6 Ind. pot $^{-1}$ 的根 瘤生物量在 $0 \mathrm{~g} \cdot \mathrm{m}^{-2} \cdot \mathrm{a}^{-1}$ 氮添加处理下与对照 $(0$ $\mathrm{g} \cdot \mathrm{m}^{-2} \cdot \mathrm{a}^{-1}, 1$ Ind. $\left.\cdot \operatorname{pot}^{-1}\right)$ 相比分别增加了 $1130 \%$ 和 $1726 \%, 5 \mathrm{~g} \cdot \mathrm{m}^{-2} \cdot \mathrm{a}^{-1}$ 氮添加处理下分别增加了 $568 \%$ 和 $43 \%$ 。同时, 氮肥和密度处理也极显著影响达乌
里胡枝子对根瘤的投资 $\left(F_{\mathrm{N}(3,48)}=63.78, F_{\mathrm{d}(2,48)}=\right.$ $21.87, p<0.001$ )(图3B)。相同密度下氮添加会显著 降低对根瘤的投资，而增加种植密度会提高植物对 根瘤的投资，从而促进根瘤的形成以及固氮。

\section{5 氮肥和种植密度对达乌里胡枝子生长和根瘤 形成的影响}

基于结构方程模型分析结果(图4), 氮肥和种植 密度通过影响叶片性状(叶片 C、N含量, 叶面积),

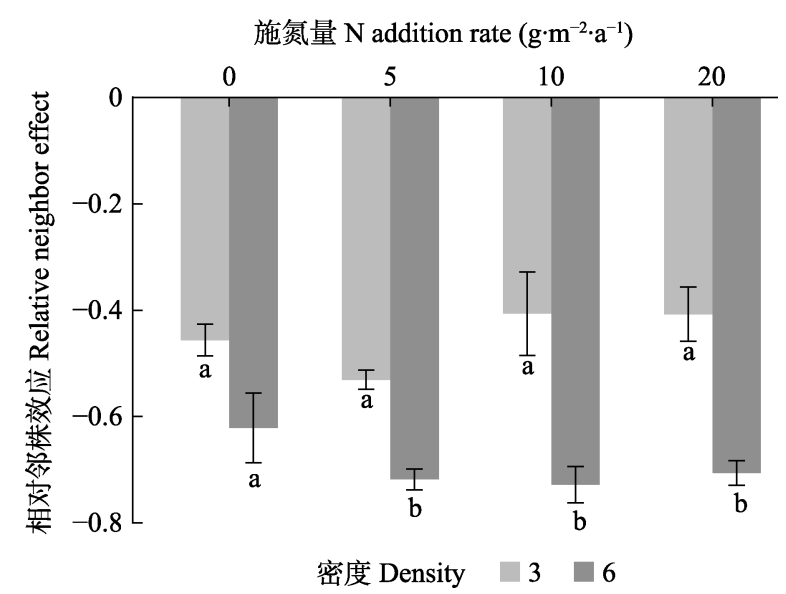

图2 不同处理下达乌里胡枝子的相对邻株效应指数(平均 值 \pm 标准误)。不同小写字母表示同一施氮量下不同密度下差 异显著 $(p<0.05)$ 。ns, $p>0.05 ; *, p<0.05 ;{ }^{* *}, p<0.01$; ***, $p$ $<0.001$ 。

Fig. 2 Relative neighbor effect index under different treatments (mean $\pm S E$ ). Different lowercase letters indicate significant difference among density treatments under the same $\mathrm{N}$ addition rate at $p<0.05$ level. ns, $p>0.05 ; *, p<0.05 ; * *, p<$ $0.01 ; * * *, p<0.001$. 


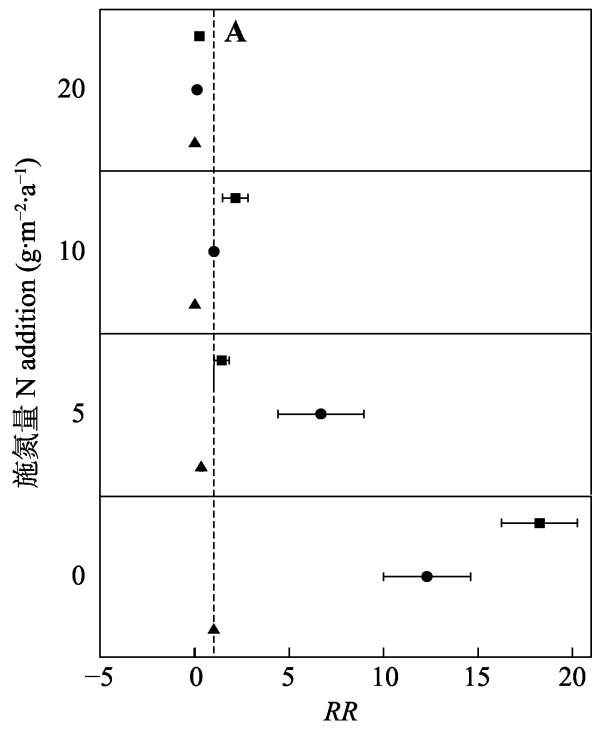

密度 Density (Ind. pot ${ }^{-1}$ )

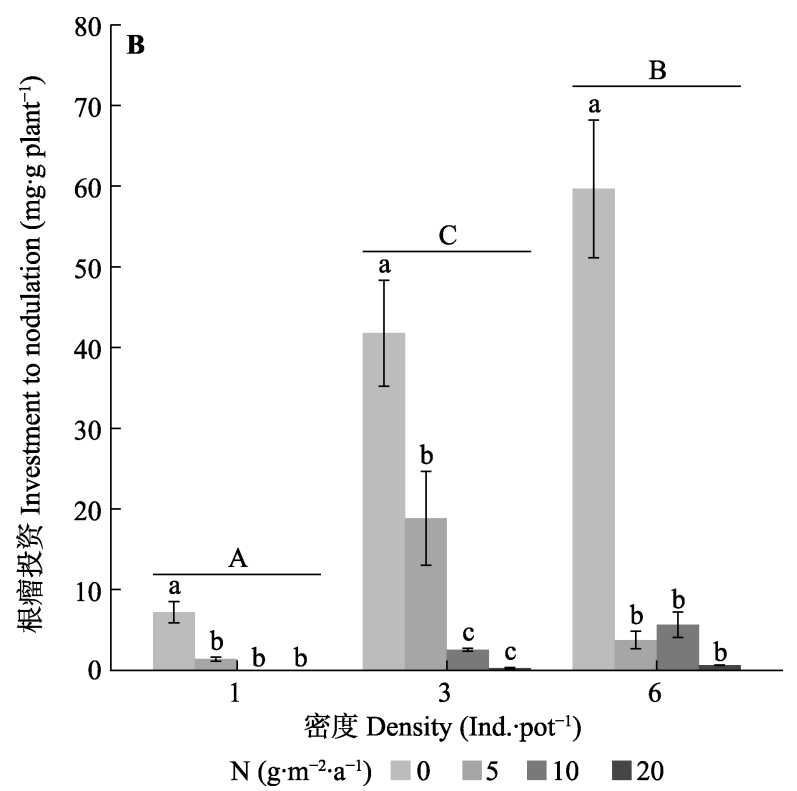

$\mathrm{N}\left(\mathrm{g} \cdot \mathrm{m}^{-2} \cdot \mathrm{a}^{-1}\right) \square 0 \quad \square 5 \quad \square 10 \quad \square 20$

图3 不同处理对根瘤生物量响应比 $(R R)(\mathbf{A})$ 和根瘤投资 $(\mathbf{B})$ 的影响(平均值 \pm 标准误)。图 $\mathbf{A}$ 中, 虚线位置代表 $R R=1$, 如果误差 线没有跨越虚线表示处理与对照存在显著差异 $(p<0.05)$ 。,$p<0.05 ; * *, p<0.01 ; * * *, p<0.001$ 。

Fig. 3 Responses of the nodule biomass response ratio $(R R)(\mathbf{A})$ and investment to nodulation $(\mathbf{B})$ under different treatments (mean \pm $S E$ ). In Fig. A, response ratio values with error bars not overlapping $R R=1$ (horizontal dotted line), indicate significant difference between treatment and control $(p<0.05){ }^{*}, p<0.05 ; * *, p<0.01 ; * * *, p<0.001$.

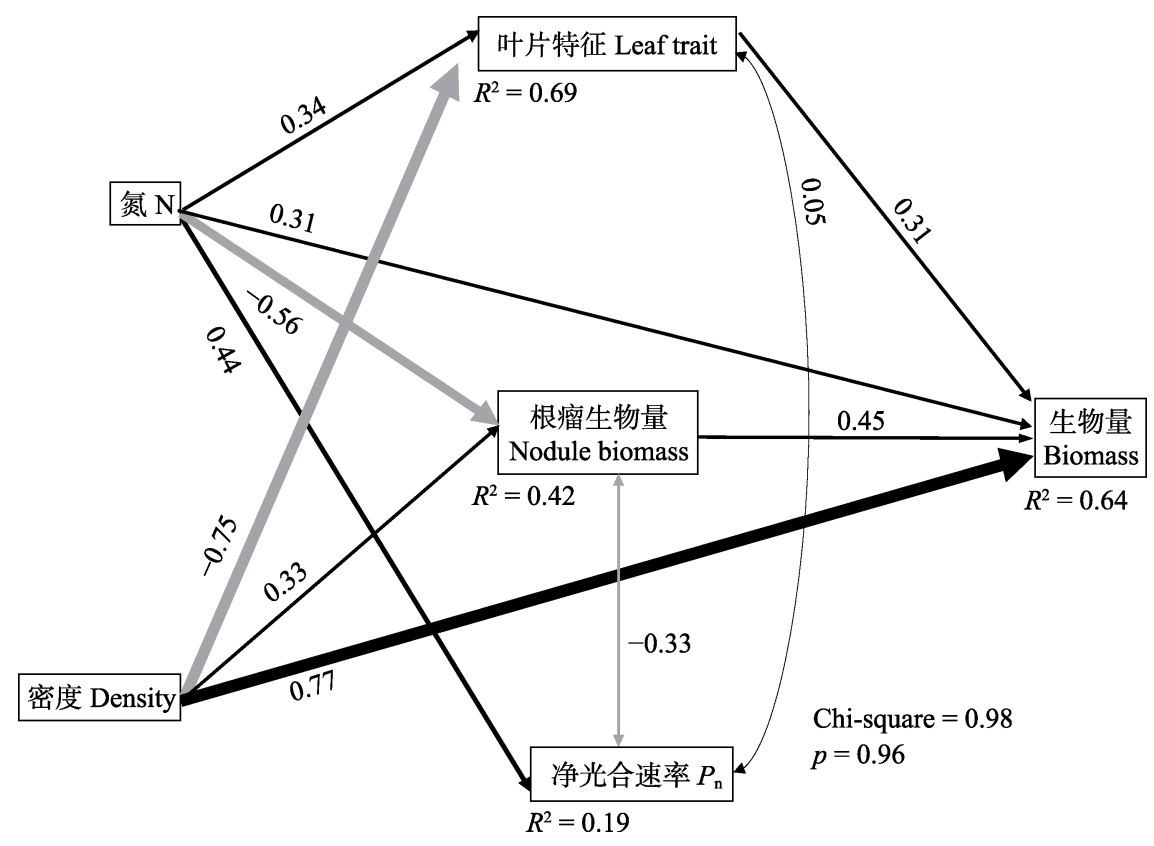

图4 氮肥和种植密度对达乌里胡枝子根瘤形成或生长的直接和间接效应。图中线条表示作用路径显著 $(p<0.05)$, 线条粗细表 示效应大小，黑色表示正效应，灰色表示负效应。 $R^{2}$ 表示解释率。

Fig. 4 Direct and indirect impacts of $\mathrm{N}$ addition rate and planting density on the root nodulation and growth of Lespedeza davurica. Lines indicate a significant effect $(p<0.05)$. Line thickness indicates relative effect size. Black lines represent positive effects, while gray lines indicate negative effects. $R^{2}$ indicates variation that can be explained. $P_{\mathrm{n}}$, net photosynthetic rate.

净光合速率以及根瘤生物量解释了达乌里胡枝子生 物量 69\%的变异。氮添加显著抑制了根瘤的形成, 路径系数为 -0.56 , 而密度对根瘤生物量的路径系数 为 0.33 , 显示正效应。

\section{3 讨论}

\section{1 氮肥和种植密度对达乌里胡枝子生长的影响} 总体来说, 添加氮肥能促进达乌里胡枝子个体 
和种群的生长, 例如总生物量增加, 叶片 $\mathrm{C} 、 \mathrm{~N}$ 含量 增加和净光合速率提高等, 这与 Tang等(2017)研究 发现氮促进草原植物生长的结果相一致。氮对植物 生长有促进作用主要是因为 $\mathrm{N}$ 是蛋白质、核酸、磷 脂等的组成成分, 同时也是叶绿素的重要组分, 与 光合作用密切相关(王忠等, 2013)。Craine等(2002) 和Humbert等(2016)发现土壤中氮的增加会降低豆 科植物在群落中的优势度和生物量, 由于本实验为 单一物种种植, 不存在物种间的竞争, 所以生物量 呈现增加的趋势。邢越(2019)也发现施氮显著提高 了单独种植的达乌里胡枝子的产量和品质。本研究

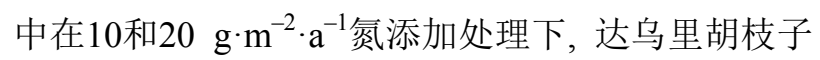
的各项生长指标均无显著差异, 说明在本实验条件 下 $10 \mathrm{~g} \cdot \mathrm{m}^{-2} \cdot \mathrm{a}^{-1}$ 的氮添加已经达到该物种生长所需的 氮阈值, 这与Bai等(2010)内蒙古草原生态系统中地 上生产力达到饱和的氮阈值 $10.5 \mathrm{~g} \cdot \mathrm{m}^{-2} \cdot \mathrm{a}^{-1}$ 的结论相 近。氮添加对达乌里胡枝子的株高虽然没有显著影 响, 但仍有增加的趋势。

种植密度对达乌里胡枝子生物量的提高主要是 通过群体产量实现的, 但对单个植株而言密度的影 响为负效应, 原因主要是因为种内竞争加剧。一方 面是较大的种植密度会导致单个植株接受的光照不 足, 影响叶片光合速率和生长发育, 净光合速率在 单株种植时大于其他密度可以说明这一点。刘文兰 等(2017)的研究同样发现随着种植密度增加, 紫苜 宿(Medicago sativa)生物量呈增加趋势, 但叶片净 光合速率、叶绿素含量则均降低; 另一方面, 密度 增加导致资源限制, 改变植物生物量分配, 影响植 物生长。本研究中相对邻株效应在 6 Ind. pot $^{-1}$ 的处 理下强于 3 Ind. pot $^{-1}$ 盆, 并且根冠比随种植密度增 加而增加, 说明达乌里胡枝子在种内竞争存在时倾 向于分配更多的生物量到地下。在 3 和 6 Ind. pot $^{-1}$ 处 理下, 达乌里胡枝子的总体产量无显著差异, 这表 明群体生物量不会随种植密度增加而一直增加, 最 终会逐渐达到饱和, 符合最终产量恒定法则 (Donald, 1951)。王荣等(2011)等研究也发现紫花苜 宿的产量随种植密度的增加呈现先增加后趋于平稳 的变化趋势。

\section{2 氮肥和种植密度对达乌里胡枝子生物固氮的 影响}

豆科植物生物固氮依赖于土壤中根瘤菌形成根 瘤, 因此根瘤生物量的一定程度上可以反映生物固
氮能力。本研究发现, 氮添加显著抑制了达乌里胡 枝子的根瘤形成, 表现在相同密度处理下达乌里胡 枝子对根瘤的投资均随氮添加量增加而呈现下降趋 势。前人的研究表明土壤中有效态氮含量是影响根 瘤菌固氮效应的重要环境因素(Malik et al., 1987)。 在根瘤菌尚未与植物建立共生关系的幼苗期, 施加 少量氮可以促进植物生长和生物固氮, 起到“起爆 氮”的作用(间艳红等, 2011; 马霞, 2013)。而在根瘤 形成后, 施加氮会降低结瘤数量和豆血红蛋白含量, 抑制固氮酶的活性, 从而影响生物固氮能力, 即产 生了“氮阻遏”的效应(Batterman et al., 2013; Regus et al., 2017; Du et al., 2020)。本实验结果中, 氮添加 可能对达乌里胡枝子的生物固氮起到了 “氮阻遏” 的 作用。

在本实验条件下, 增加种植密度对根瘤形成有 一定的促进作用, 这主要是因为随着种植密度的增 加, 植株内竞争加剧, 导致单个植物获得的资源减 少。这从叶片 $\mathrm{N}$ 含量和单株生物量随密度增加而降 低可以发现, 因此推测达乌里胡枝子会一定程度发 展根瘤固氮来弥补氮供应不足的情况。另外, 与单 株种植相比, 另外两种种植密度在所有施氮梯度下 均有根瘤产生，说明增加种植密度对施氮后的“氮 阻遏”有减缓效应。陈红卫(2015)对大豆/玉米(Zea mays)间作系统的研究发现, 玉米种植密度的增加 使根系对氮素竞争加剧, 从而导致大豆根系的根瘤 数量和固氮能力增加。Kapustka和Wilson (1990)发 现增加大豆种植密度会提高固氮酶的活性但降低结 瘤数。此外, 本研究中发现施氮量为 $10 \mathrm{~g} \cdot \mathrm{m}^{-2} \cdot \mathrm{a}^{-1}$ 种 植密度为 $3 \mathrm{Ind} . \mathrm{pot}^{-1}$ (约 96 株 $\cdot \mathrm{m}^{-2}$ ), 或施氮量为 5 $\mathrm{g} \cdot \mathrm{m}^{-2} \cdot \mathrm{a}^{-1}$ 种植密度为 6 Ind. $\operatorname{pot}^{-1}$ (约 192 株 $\cdot \mathrm{m}^{-2}$ ) 时, 达乌里胡枝子对根瘤的投资和根瘤生物量都维持在 较高的水平, 总生物量与对照相比分别增加 $165 \%$ 和 $169 \%$, 并且与施氮量 $20 \mathrm{~g} \cdot \mathrm{m}^{-2} \cdot \mathrm{a}^{-1}$ 处理无显著差 异 $(p>0.05)$, 说明在本控制实验中, 这两种处理能 最大程度发挥“施氮增产”的作用和缓解“氮阻遏”。

\section{4 结论}

本研究发现, 土壤氮供给和植物种植密度共同 调节了达乌里胡枝子的生长 (二者解释变化的 $64 \%$ ) 和生物固氮能力(解释变化的 $42 \%$ )。氮肥和种植密度 对达乌里胡枝子的种群产量均有促进作用, 氮肥提 高了达乌里胡枝子叶片的 $\mathrm{C} 、 \mathrm{~N}$ 含量和净光合速率, 
密度增加会导致达乌里胡枝子的种内竞争加剧, 降 低叶片 $\mathrm{N}$ 含量和净光合速率, 但由于群体数量增多 会促进植物产量增加。氮肥会降低达乌里胡枝子对 根瘤的投资和根瘤生物量, 从而抑制达乌里胡枝子 的生物固氮, 密度增加导致单个植株资源受限, 因 此会促进根瘤的形成来固定更多的大气氮, 密度增 加会缓解氮肥带来的“氮阻遏”。该实验条件下, 达 乌里胡枝子产量达到稳定时的氮添加量为 10 $\mathrm{g} \cdot \mathrm{m}^{-2} \cdot \mathrm{a}^{-1}$ 。当施氮量为 $10 \mathrm{~g} \cdot \mathrm{m}^{-2} \cdot \mathrm{a}^{-1}$ 种植密度为 3 Ind. $\operatorname{pot}^{-1}$ (约 96 株 $\cdot \mathrm{m}^{-2}$ ), 或施氮量为 $5 \mathrm{~g} \cdot \mathrm{m}^{-2} \cdot \mathrm{a}^{-1}$ 种植 密度为6 Ind. pot $^{-1}$ (约192株. $\mathrm{m}^{-2}$ ) 时能最大程度发挥 “施氮增产”和种植密度缓解“氮阻遏”作用。本研究 结果为干旱、半干旱区天然退化草地的恢复以及人 工种植豆科作物草地的管理提供了理论依据, 但温 室条件下难以反映真实的自然情况, 因此实际情况 下的氮添加量和种植密度仍需进一步研究。

致谢 感谢山西农业大学牧站草学实验室提供的实 验材料以及各位老师的帮助。

\section{参考文献}

Bai YF, Wu JG, Clark CM, Naeem S, Pan QM, Huang JH, Zhang LX, Han XG (2010). Tradeoffs and thresholds in the effects of nitrogen addition on biodiversity and ecosystem functioning: evidence from Inner Mongolia grasslands. Global Change Biology, 16, 358-372.

Batterman SA, Wurzburger N, Hedin LO (2013). Nitrogen and phosphorus interact to control tropical symbiotic $\mathrm{N}_{2}$ fixation: a test in Inga punctata. Journal of Ecology, 101, 1400-1408.

Chen HW (2015). Regulatory Mechanism of Interspecific $N$ Compensatory Utilization by Plant Density in Maize/Soybean Intercropping Systems. PhD dissertation, Gansu Agricultural University, Lanzhou. 40-49. [陈红卫 (2015). 玉米/大豆间作氮素补偿利用的密度调控机理. 博士学位论文, 甘肃农业大学, 兰州. 40-49.]

Chen MJ, Li CL, Qi Y (1997). Studies on biological features of Lespedeza and its nutrient values. Natural Resources, 19(2), 74-81. [陈默君, 李昌林, 祁永 (1997). 胡枝子生 物学特性和营养价值研究. 自然资源, 19(2), 74-81.]

Cheng J, Cheng JM, Hu TM (2011). Distribution responses of Lespedeza davurica community on Loess Plateau to climate change. Chinese Journal of Applied Ecology, 22, 35-40. [程杰, 程积民, 呼天明 (2011). 气候变化对黄土 高原达乌里胡枝子种群分布格局的影响. 应用生态学 报, 22, 35-40.]

Chu CJ, Maestre FT, Xiao S, Weiner J, Wang YS, Duan ZH, Wang G (2008). Balance between facilitation and resource competition determines biomass-density relationships in plant populations. Ecology Letters, 11, 1189-1197.

Craine JM, Tilman D, Wedin D, Reich P, Tjoelker M, Knops J (2002). Functional traits, productivity and effects on nitrogen cycling of 33 grassland species. Functional Ecology, 16, 563-574.

Diao LW, Li P, Liu WX, Xu S, Qiao CL, Zeng H, Liu LL (2018). Response of plant biomass to nitrogen addition and precipitation increasing under different climate conditions and time scales in grassland. Chinese Journal of Plant Ecology, 42, 818-830. [ᄀ励玮, 李平, 刘卫星, 徐㚳, 乔 春连, 曾辉, 刘玲莉 (2018). 草地生态系统生物量在不 同气候及多时间尺度上对氮添加和增雨处理的响应. 植物生态学报, 42, 818-830.]

Donald CM (1951). Competition among pasture plants. I. Intraspecific competition among annual pasture plants. Australian Journal of Agricultural Research, 2, 355-376.

Drake DC (2011). Invasive legumes fix $\mathrm{N}_{2}$ at high rates in riparian areas of an N-saturated, agricultural catchment. Journal of Ecology, 99, 515-523.

Du MK, Gao Z, Li XX, Liao H (2020). Excess nitrate induces nodule greening and reduces transcript and protein expression levels of soybean leghaemoglobins. Annals of Botany, 126, 61-72.

Du RF (2012). The Response of Antioxidant Protection System of Lespedeza davurica to the Combined Stress of Drought and UV-B Radiation. Master degree dissertation, Northwest Agriculture \& Forestry University, Yangling, Shaanxi. 13-24. [杜润峰 (2012). 达乌里胡枝子抗氧化 防御系统对干旱、UV-B辐射及复合胁迫的动态响应. 硕士学位论文, 西北农林科技大学, 陕西杨凌. 13-24.]

Duan DP, Xu BC, Niu FR, Xu WZ (2012). Effects of water and phosphorus on chlorophyll fluorescence characteristics of different position leaves in Lespedeza daurica. Pratacultural Science, 29, 422-428. [段东平, 徐炳成, 牛富荣, 徐 伟洲 (2012). 水分和磷肥对达乌里胡枝子不同叶位叶 绿素苂光参数特征的影响. 草业科学, 29, 422-428.]

Elser JJ, Bracken ME, Cleland EE, Gruner DS, Harpole WS, Hillebrand H, Ngai JT, Seabloom EW, Shurin JB, Smith JE (2007). Global analysis of nitrogen and phosphorus limitation of primary producers in freshwater, marine and terrestrial ecosystems. Ecology Letters, 10, 1135-1142.

Galloway JN, Dentener FJ, Capone DG, Boyer EW, Howarth RW, Seitzinger SP, Asner GP, Cleveland CC, Green PA, Holland EA, Karl DM, Michaels AF, Porter JH, Townsend AR, Vorosmarty CJ (2004). Nitrogen cycles: past, present, and future. Biogeochemistry, 70, 153-226.

Galloway JN, Townsend AR, Erisman JW, Bekunda M, Cai Z, Freney JR, Martinelli LA, Seitzinger SP, Sutton MA (2008). Transformation of the nitrogen cycle: recent trends, questions, and potential solutions. Science, 320, 889-892.

www.plant-ecology.com 
Guan SB (2017). Effect of Fertilization and Planting Density on Seed Yield of Lespedeza davurica. Master degree dissertation, Shanxi Agricultural University, Taigu, Shanxi. 10-11. [关少波 (2017). 施肥和种植密度对达乌里胡枝子种子 产量的影响. 硕士学位论文, 山西农业大学, 山西太谷. 10-11.]

Guinet M, Nicolardot B, Revellin C, Durey V, Carlsson G, Voisin AS (2018). Comparative effect of inorganic $\mathrm{N}$ on plant growth and $\mathrm{N}_{2}$ fixation of ten legume crops: towards a better understanding of the differential response among species. Plant and Soil, 432, 207-227.

Gutschick VP (1981). Evolved strategies in nitrogen acquisition by plants. The American Naturalist, 118, 607-637.

Humbert JY, Dwyer JM, Andrey A, Arlettaz R (2016). Impacts of nitrogen addition on plant biodiversity in mountain grasslands depend on dose, application duration and climate: a systematic review. Global Change Biology, 22, 110-120.

Japhet W, Zhou DW, Zhang HX, Zhang HX, Yu T (2009). Evidence of phenotypic plasticity in the response of Fagopyrum esculentum to population density and sowing date. Journal of Plant Biology, 52, 303-311.

Kapustka LA, Wilson KG (1990). The influence of soybean planting density on dinitrogen fixation and yield. Plant and Soil, 129, 145-156.

LeBauer DS, Treseder KK (2008). Nitrogen limitation of net primary productivity in terrestrial ecosystems is globally distributed. Ecology, 89, 371-379.

Lie ZY, Xu SK, Xue L, Huang WL, Li J (2016). Effect of density on the growth of evergreen Tephrosia candida shrubs. Journal of Southwest Forestry University, 36, 69-73. [列 志昒, 许松葵, 薛立, 黄威龙, 李洁 (2016). 密度对山 毛豆幼林生长的影响. 西南林业大学学报, 36, 69-73.]

Liu WL, Shi SL, Tian FP (2017). Effects of planting density on biomass and photosynthetic characters of alfalfa leaves at different positions. Grassland and Turf, 37, 14-19. [刘文 兰, 师尚礼, 田福平 (2017). 种植密度对紫花苜宿生物 量与不同叶位光合特性的影响. 草原与草坪, 37, 14-19.]

Liu XJ, Zhang Y, Han WX, Tang AH, Shen JL, Cui ZL, Vitousek P, Erisman JW, Goulding K, Christie P, Fangmeier A, Zhang FS (2013). Enhanced nitrogen deposition over China. Nature, 494, 459-462.

Ma X, Wang LL, Li WJ, Song JP, He Y, Luo M (2013). Effects of different nitrogen levels on nitrogen fixation and seed production of alfalfa inoculated with rhizobia. Acta Prataculturae Sinica, 22, 95-102. [马霞, 王丽丽, 李卫军, 宋江平, 何媛, 罗明 (2013). 不同施氮水平下接种根瘤 菌对苜宿固氮效能及种子生产的影响. 草业学报, 22, 95-102.]

Ma YJ, Cao ZZ, Li Y (2010). Advances in basic theroy of Lespedeza spp. Pratacultural Science, 27, 128-134. [马彦军, 曹致中, 李毅 (2010). 胡枝子属植物研究进展. 草业科
学, 27, 128-134.]

Malik NS, Calvert HE, Bauer WD (1987). Nitrate induced regulation of nodule formation in soybean. Plant Physiology, 84, 266-271.

Menge DNL, Levin SA, Hedin LO (2009). Facultative versus obligate nitrogen fixation strategies and their ecosystem consequences. The American Naturalist, 174, 465-477.

Midolo G, Alkemade R, Schipper AM, Benítez-López A, Perring MP, de Vries W (2019). Impacts of nitrogen addition on plant species richness and abundance: a global meta-analysis. Global Ecology and Biogeography, 28, 398-413.

Pan QM, Bai YF, Han XG, Yang JC (2005). Effects of nitrogen additions on a Leymus chinensis population in typical steppe of Inner Mongolia. Acta Phytoecologica Sinica, 29, 311-317. [潘庆民，白永飞, 韩兴国，杨景成 (2005). 氮 素对内蒙古典型草原羊草种群的影响. 植物生态学报, 29, 311-317.]

Pardo LH, Fenn ME, Goodale CL, Geiser LH, Driscoll CT, Allen EB, Baron JS, Bobbink R, Bowman WD, Clark CM, Emmett B, Gilliam FS, Greaver TL, Hall SJ, Lilleskov EA, et al. (2011). Effects of nitrogen deposition and empirical nitrogen critical loads for ecoregions of the United States. Ecological Applications, 21, 3049-3082.

Regus JU, Wendlandt CE, Bantay RM, Gano-Cohen KA, Gleason NJ, Hollowell AC, O’Neill MR, Shahin KK, Sachs JL (2017). Nitrogen deposition decreases the benefits of symbiosis in a native legume. Plant and Soil, 414, 159-170.

Tang ZS, Deng L, An H, Yan WM, Shangguan ZP (2017). The effect of nitrogen addition on community structure and productivity in grasslands: a meta-analysis. Ecological Engineering, 99, 31-38.

Vitousek P, Howarth R (1991). Nitrogen limitation on land and in the sea: How can it occur? Biogeochemistry, 13, 87-115.

Vitousek PM, Menge DNL, Reed SC, Cleveland CC (2013). Biological nitrogen fixation: rates, patterns and ecological controls in terrestrial ecosystems. Philosophical Transactions of the Royal Society B: Biological Sciences, 368, 20130119. DOI: 10.1098/rstb.2013.0119.

Wang Y, Duan XY, Zhang SC, Duan XL, Yang HL, Qian BL (2011). Influence of planting density on grass yield and other biological properties of Medicago sativa. Prataultural Science, 28, 1400-1402. [王芗, 段学义, 张胜昌, 段 晓丽, 杨慧玲, 钱宝玲 (2011). 紫花苜宿播种密度对草 产量及其他生物学性状的影响. 草业科学, 28, 1400-1402.]

Wang Z (2013). Plant Physiology. 2nd ed. China Agriculture Press, Beijing. 80-119. [王忠 (2013). 植物生理学. 二版. 中国农业出版社, 北京. 80-119.]

Weiner J, Stoll P, Muller-Landau H, Jasentuliyana A (2001). The effects of density, spatial pattern, and competitive 
symmetry on size variation in simulated plant populations. The American Naturalist, 158, 438-450.

Xing Y (2019). Effects of Nitrogen Annlication on the Yield, Quauality and Bud Bank of Mixed Planting of Leymus chinensis and Lespedeza daurica. Master degree dissertation, Northeast Normal University, Changchun. 13-20. [邢 越 (2019). 施氮对羊草和胡枝子混播种植的产量和品 质及芽库的影响. 硕士学位论文, 东北师范大学, 长春. 13-20.]

Yan YH, Yang WY, Zhang XQ, Chen XL, Chen ZQ (2011). Effects of different nitrogen levels on photosynthetic characteristics, dry matter accumulation and yield of relay strip intercropping Glycine max after blooming. Acta Prataculturae Sinica, 20，233-238. [间艳红, 杨文钰, 张新 全, 陈小林, 陈忠群 (2011). 施氮量对套作大豆花后光 合特性、干物质积累及产量的影响. 草业学报, 20 , 233-238.]

Yang JX, Huang SS, Yang ML, Wang JA (2012). Effect of density and fertilizer amount on yield of different branching types of soybeans. Soybean Science, 31, 381-384. [杨 继学, 黄珊珊, 杨明亮, 王继安 (2012). 密度和施肥量 对不同分枝类型大豆产量的影响. 大豆科学, 31, 381-384.]

Yu GR, Jia YL, He NP, Zhu JX, Chen Z, Wang QF, Piao SL, Liu XJ, He HL, Guo XB, Wen Z, Li P, Ding GA, Goulding $K$ (2019). Stabilization of atmospheric nitrogen deposition in China over the past decade. Nature Geoscience, $12,424-429$.

Zhang MC, Zhan YC, He SY, Jin XJ, Wang MX, Ren CY, Zhang YX (2018). Effects of different nitrogen fertilizer and density level on dry matter accumulation and yield of adzuki bean. Crops, 34, 141-146. [张明联, 战英策, 何松 榆, 金喜军, 王孟雪, 任春元, 张玉先 (2018). 氮密交 互对红小豆干物质积累规律及产量的影响. 作物杂志, 34, 141-146.]

Zhang WP, Wang GX (2010). Positive interactions in plant communities. Acta Ecologica Sinica, 30, 5371-5380. [张 炜平, 王根轩 (2010). 植物邻体间的正相互作用. 生态 学报, 30, 5371-5380.]

Zhang XH, Xu BC, Li FM (2007). Effect of planting density on the productivity and WUE of three legumes in highland of Loess Plateau. Acta Agrestia Sinica, 15, 593-598. [张晓红, 徐炳成, 李凤民 (2007). 密度对三种豆科牧草生产力和 水分利用率的影响. 草地学报, 15, 593-598.]

Zhao HK, Ma Z, Zhang CH, Lei ZL, Yao BQ, Zhou HK (2016). The reproductive allocation of Avena sativa under different planting densities and nitrogen addition treatments. Pratacultural Science, 33, 249-258. [赵宏鬼, 马 真, 张春辉, 雷占兰, 姚步青, 周华坤 (2016). 种植密 度和施氮水平对燕麦生物量分配的影响. 草业科学, 33, 249-258.]

Zhao X, Dong KH, Zhang Y, Yang WD, Liang PF (2009). Study on Lamina anatomical structure of Lespedeza daurica (Laxm.) Schindl. from different populations. Acta Agrestia Sinica, 17, 445-451. [赵祥, 董宽虎, 张圭, 杨武 德, 梁丕富 (2009). 不同居群达乌里胡枝子叶片解剖结 构研究. 草地学报, 17, 445-451.]

责任编委: 邓建明 责任编辑: 李 敏 